\title{
Gauge fixing for logarithmic connections over curves and the Riemann-Hilbert-Problem*
}

\author{
Christian Gantz ${ }^{\dagger}$ and Brian Steer \\ Mathematical Institute, Oxford OX1 3LB, UK \\ (gantz@maths.ox.ac.uk)
}

November 2, 2018

\section{Contents}

\begin{tabular}{lll}
\hline 1 & Introduction & 2
\end{tabular}

2 Local logarithmic connections and weighted flat bundles 5

2.1 Logarithmic connections . . . . . . . . . . . . . 5

$2.2 \quad$ Gauge fixing for logarithmic connections . . . . . . . . . . 7

2.3 Correspondence between local logarithmic connections and weighted flat bundles . . . . . . . . . . . . . . . 11

3 Global logarithmic connections and weighted flat bundles 14

$3.1 \quad$ Correspondence over $\mathbb{P}^{1} \ldots \ldots \ldots \ldots \ldots 14 \ldots \ldots \ldots$

3.2 The splitting type of $E \rightarrow \mathbb{P}^{1} \ldots \ldots \ldots \ldots$

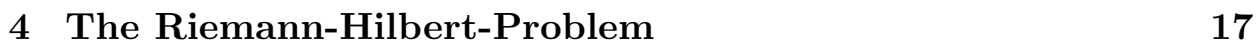

4.1 Commutative and semi-simple representations . . . . . . . . . 17

4.2 The rank two case $\ldots \ldots \ldots \ldots \ldots$

4.3 The semi-stable case and implications . . . . . . . . . . . 21

4.4 Reducible representations . . . . . . . . . . . . . . . . . 23

$4.5 \quad$ Parabolic representations and the rank three case . . . . . . . 25

${ }^{*}$ Mathematics Subject Classification (1991) 14H60 (Primary) 14H30 14F10 14F35 (Secondary)

${ }^{\dagger}$ The first author was fully supported by the Alfried Krupp von Bohlen und Halbach Stiftung, Essen 


\section{Introduction}

SAID BRIEFLY: We explain in detail the correspondence $\mathcal{F}$ between algebraic connections over $\mathbb{P}^{1}$, logarithmic at $X=\left\{x_{1}, \ldots, x_{n}\right\} \subseteq \mathbb{P}^{1}$, and flat bundles over $\mathbb{P}^{1}-X$ with integer weighted filtrations near each $x_{j}$. Included is a gauge fixing theorem for logarithmic connections. (Thus far, one could work over any Riemann surface.) We prove a bound on the splitting type of a semistable logarithmic connection over $\mathbb{P}^{1}$. Using this we extend some results on the Riemann-Hilbert-Problem and explain some others. The work is self contained and elementary, using only basic knowledge of gauge theory and the Birkhoff-Grothendieck-Theorem.

The CONCEPTs: A logarithmic connection over $\left(\mathbb{P}^{1}, X\right)$ consists of a holomorphic vector bundle $E \rightarrow \mathbb{P}^{1}$ with an algebraic connection

$$
\nabla: \Omega^{0}(E) \rightarrow \Omega^{0}(E) \otimes \Omega_{\mathbb{P}^{1}}^{1}(\log X)
$$

satisfying the Leibnitz rule, where $\Omega_{\mathbb{P}^{1}}^{1}(\log X)$ is the sheaf of holomorphic 1 -forms generated near $x_{j}$ by $\mathrm{d} z_{j} / z_{j}$ for a coordinate $z_{j}$ centred at $x_{j} . H:=$ $\left.(E, \nabla)\right|_{\mathbb{P}^{1}-X}$ is a flat bundle. Isomorphism classes of flat bundles of rank $r$ correspond to conjugacy classes of representations $\chi: \pi_{1}\left(\mathbb{P}^{1}-X\right) \rightarrow$ $\mathrm{Gl}(r, \mathbb{C})$, [2, p 200], [1], p 51-56], [13, p 4]. $\chi$ is called the monodromy (or holonomy) of $H$.

If $E$ is trivial, one calls $(E, \nabla)$ a Fuchsian system. Choose a global coordinate $z$ on $\mathbb{P}^{1}$ such that $a_{j}:=z\left(x_{j}\right) \neq \infty$. For any Fuchsian system $\left(\mathbb{P}^{1} \times \mathbb{C}^{r}, \nabla\right)$ there exist $B_{j} \in$ End $\left(\mathbb{C}^{r}\right)$, [1, p 4$]$, such that

$$
\nabla=\mathrm{d}+\sum_{j=1}^{n} \frac{B_{j}}{z-a_{j}} .
$$

The PRoblem: In 1900 Hilbert stated his twenty first problem: Prove that for any given singularities $X$ and representation $\chi$ there exists a Fuchsian system realising $(X, \chi)$. Literally, [12], he said Fuchsian equation, i.e. higher order differential equations with prescribed singularities. But Anosov \& Bolibruch argue that he meant vector-valued linear equations, i.e. Fuchsian systems, because the alternative was already known to be wrong in 1900 . Fuchsian equations induce Fuchsian systems, [1, Ch. 7]. Since Riemann worked on the problem earlier, it is called the Riemann-Hilbert-Problem (RHP). For a comprehensive collection of known results and references to 
the RHP see [1], also [3] and [4]. Much of the recent work is due to Bolibruch. (An approach different from most is Hain's, [11].) Bolibruch discovered a pair $(X, \chi)$, of rank $r=3$ and with $n=4$, which cannot be realised by any Fuchsian system, [4, p 74-76], [1, p 14]. Therefore, he modified the RHP to the question of which $(X, \chi)$ can occur on Fuchsian systems. Bolibruch shows that for fixed $\chi$ but varying $X$, the answer can be different. We do not consider the dependence on $X$ and concentrate on positive answers to the RHP.

By the Birkhoff-Grothendieck-Theorem (BGT), [18], any vector bundle $E \rightarrow \mathbb{P}^{1}$ is isomorphic to $\mathcal{O}\left(c_{1}\right) \oplus \ldots \oplus \mathcal{O}\left(c_{r}\right)$ for unique integers $c_{1} \geq \ldots \geq c_{r}$, called the splitting type of $E$. So, considering the space of all logarithmic connections over $\left(\mathbb{P}^{1}, X\right)$, the Fuchsian systems (1) constitute the connected component of the trivial connection. Fuchsian systems are clearly semistable.

The Approach: We follow Deligne, [6]. To each $x_{j}$, let $U_{j}$ be a small simply-connected neighbourhood and $U_{j}^{*}:=U_{j}-\left\{x_{j}\right\}$. We show directly that a logarithmic connection $(E, \nabla)$ admits, over a small open neighbourhood of $x_{j}$, a normal trivialisation (Definition 2.2.1). This is used to construct on $H=\left.(E, \nabla)\right|_{\mathbb{P}^{1}-X}$ a filtration $0 \subset H_{j}^{1} \subset \ldots \subset H_{j}^{l_{j}}=\left.H\right|_{U_{j}^{*}}$ by flat subbundles with integer weights $\Phi_{j}=\operatorname{diag}\left(\phi_{j}^{i}\right),\left(\phi_{j}^{1} \geq \ldots \geq \phi_{j}^{r}\right) \in \mathbb{Z}^{r}$, for each $j=1, \ldots, n$. Conversely, such data on a flat bundle $H \rightarrow\left(\mathbb{P}^{1}-X\right)$ induces a unique extension of $H$ to a logarithmic connection $(E, \nabla):=$ $\mathcal{F}\left(H, H_{j}^{m}, \Phi_{j}\right)$ over $\left(\mathbb{P}^{1}, X\right)$.

Extending and restricting appropriate morphisms, $\mathcal{F}$ becomes an equivalence between the categories of weighted flat bundles $\left(H, H_{j}^{m}, \Phi_{j}\right)$ over $\mathbb{P}^{1}-X$ and the category of logarithmic connections $(E, \nabla) \rightarrow\left(\mathbb{P}^{1}, X\right)$. The equivalence $\mathcal{F}$ has been constructed slightly differently by Manin, [16]; Deligne, [6], and Simpson, [21], and on objects partially by Anosov \& Bolibruch, see also [8]. $\mathcal{F}$ prerves injections and surjections. The integer weights are used to define the degree of a weighted flat bundle. By Simpson, $\mathcal{F}$ preserves degrees and hence (semi-) stability (Definition 3.1.2).

If $\gamma_{j}$ is a loop in $U_{j}^{*}$ going once around $x_{j}$, the parallel transport in $H$ w.r.t. $\gamma_{j}$ is conjugation equivalent to an upper-triangular matrix. So, filtrations of $\left.H\right|_{U_{j}^{*}}$ by flat subbundles exist. There is much freedom in choosing integer weights. Hence, any pair $(X, \chi)$ is realized by several logarithmic connections. (This even holds over Riemann surfaces, [20.) If one is satisfied with any logarithmic connection realizing a given pair $(X, \chi)$, the problem 
is therefore solved; the difficulty is to decide when the underlying bundle is trivial.

We seek, for given $\mathrm{H}$, filtrations $H_{j}^{m}$ and integer weights $\Phi_{j}$ such that $\mathcal{F}\left(H, H_{j}^{m}, \Phi_{j}\right)$ is Fuchsian. To indicate the relation between our approach and previous ones, let $(X, \chi)$ be realised by $(E, \nabla)$. $E$ admits a system $W=\left(w_{1}, \ldots, w_{r}\right)$ of global meromorphic section, holomorphic away from $x_{1}$, spanning $E$ off $x_{1}$. $W$ generates a flat bundle over $\mathbb{P}^{1}$. So, every pair $(X, \chi)$ is realized by a regular system, i.e. a singular algebraic connection on $\mathbb{P}^{1} \times \mathbb{C}^{r}$ such that the flat sections have at most polynomial growth. This has long been known, [19], [6], and most attempts to find Fuchsian systems are by "modifying" (see [1, p 77]) regular ones. Conversely, regular systems induce logarithmic connections. To see this, use the system of sections $V$ as in equation (2.2.21) of [1] to generate a free rank $r$ sheaf, i.e. vector bundle, and apply Levelt's result, [1, p 28], [15, p 379]. The modification of regular systems does correspond to changing filtrations and integer weights on $H$. Bolibruch essentially introduced the approach, but worked himself mainly via regular systems.

Bolibruch found that any irreducible representation is the monodromy of a Fuchsian system for any given singularities, [1, p 83]. Having this, one attempts to apply induction on reducible ones. The difficulty is that the smaller subspaces in the local filtrations of $H$ have higher integer weights and tend to be contained in global subspaces. This restricts the choice of filtrations and weights which make $H$ into a semi-stable weighted flat bundle; which is neccessary should $H$ be a restriction of a Fuchsian system. This difficulty comes up in Theorems 4.4.1 and 4.5.1 and, in an extreme form in Proposition 4.4.2. The results on reducible representations that we have, Lemma 3.2.4, follow from the preservation of short exact sequences under $\mathcal{F}$ and the BGT.

WHAT IS NEW: We give a direct proof of a gauge fixing theorem for logarithmic connections over curves, Theorem 2.2.3. The description of the inverse of $\mathcal{F}$ via this gauge fixing theorem seems new.

We work on the RHP via logarithmic connections, avoiding regular systems. Instead of Bolibruch's sum of exponents of a regular system we use the degree of a bundle over $\mathbb{P}^{1}$ and the concept of semi-stability. In particular, the preservation of semi-stability under $\mathcal{F}$ is usefull because any Fuchsian system is semi-stable. Bolibruch does not mention the concept of semistability in relation to the RHP. Applying the properties of $\mathcal{F}$, explained in the first part of this article, several of Bolibruch's results on the RHP follow 
easily from the Birkhoff-Grothendieck-Theorem and the fact that $\mathrm{H}^{0}(\mathcal{O}(c))$ equals 0 if $c<0$ and $\mathbb{C}$ if $c=0$. We do not reprove this way as many results as possible, restricting to some signific ones, e.g. Theorem 4.2.1, Proposition 4.4.2, Lemma 3.2.4 here and [1, Lem. 5.2.2] and [1, Thm. 5.2.2].

Besides, perhaps, a more conceptual proof of known results, we have new ones. Bolibruch's first counter-example to the RHP implies that a semistable logarithmic connection is not neccessarily Fuchsian. However, we prove that any semi-stable logarithmic connection $(E, \nabla)$ has bounded splitting type, Theorem 3.2.1. To be precise, $c_{i}-c_{i+1} \leq n-2$ for $i=1, \ldots, r-1$ where $E=\mathcal{O}\left(c_{1}\right) \oplus \ldots \oplus \mathcal{O}\left(c_{r}\right), c_{1} \geq \ldots \geq c_{r}, n=\sharp X$. Bolibruch treats the special case of logarithmic connections with irreducible monodromy. His bound, Corollary 3.2.2 here, is weaker. Combined with a technical result of Bolibruch, namely Proposition 4.1.2 here, i.e. [1], Lem. 4.1.3], of which we provide a direct proof, our bound leads to the existence of a Fuchsian system with given monodromy $\chi: \pi_{1}\left(\mathbb{P}^{1}-X\right) \rightarrow \operatorname{Aut}\left(\mathbb{C}^{r}\right)$ if some $\chi\left(\gamma_{k}\right)$ admits an eigenvector which is a cyclic vector of the $\pi_{1}\left(\mathbb{P}^{1}-X\right)$-module $\mathbb{C}^{r}$. Firstly, this implies Bolibruch's positive solution for irreducible representations. Secondly, it gives a shorter proof of his result that each $\chi$ is a subrepresentation of the monodromy of a Fuchsian system of double the rank. Thirdly, it leads to an alternative proof of his complete answer to the RHP in rank three.

We have a new, sufficient condition for parabolic representations to come from Fuchsian systems, Theorem 4.5.1, and show that this is always satisfied in rank four. A new result for reducible representations is part (ii) of Theorem 4.4.1.

Acknowledgements: We thank Michael Thaddeus for bringing the RHP to our attention and showing us Bolibruch's work.

\section{Local logarithmic connections and weighted flat bundles}

\subsection{Logarithmic connections}

Let $U$ be a simply connected, open neighbourhood of $0 \in \mathbb{C}, z$ the natural coordinate. By $\Omega_{U}^{p}$ we denote the sheaves of holomorphic forms on $U$, Let

$$
\Omega_{U}^{1}(\log 0):=\Omega_{U}^{0} \cdot\left(\frac{\mathrm{d} z}{z}\right)
$$


be the free sheaf of holomorphic 1-forms logarithmic at 0, [10, p 449]. Naturally, this sheaf is isomorphic to $\Omega_{U}^{0}\left(\mathcal{K}_{U} \otimes[0]\right)$, where $\mathcal{K}_{U}$ is the canonical bundle with section $\mathrm{d} z$ and $[0]$ the line bundle on $U$ associated with the divisor 0 . The vector $(\mathrm{d} z / z)(0) \in\left(\mathcal{K}_{U} \otimes[0]\right)_{0}$ is independent of the choice of coordinate because, if $u: U \rightarrow \mathbb{C}$ is another coordinate with $u(0)=0$,

$$
\frac{\mathrm{d} u}{u}=\frac{z \mathrm{~d} u}{\mathrm{~d} z} \cdot \frac{\mathrm{d} z}{z}=(1+o(z)) \frac{\mathrm{d} z}{z} .
$$

Definition 2.1.1 A (local) connection logarithmic at 0 is a holomorphic vector bundle $E \rightarrow U$ and a $\mathbb{C}$-linear map

$$
\nabla: \Omega^{0}(E) \rightarrow \Omega^{0}(E) \otimes \Omega_{U}^{1}(\log 0)
$$

satisfying the Leibnitz rule $\nabla(f v)=(d f) v+f \nabla(v)$ for all $f \in \Omega^{0}$ and $v \in \Omega^{0}(E)$. A morphism $\tau:\left(E^{\prime}, \nabla^{\prime}\right) \rightarrow(E, \nabla)$ of logarithmic connections is a bundle map such that

$$
\tau_{*} \circ \nabla^{\prime}=\nabla \circ \tau_{*}
$$

for $\tau_{*}: \Omega^{0}\left(E^{\prime}\right) \rightarrow \Omega^{0}(E)$.

A morphism is called injective (surjective) if it is as bundle map. A short sequence $\left(E^{\prime}, \nabla^{\prime}\right) \rightarrow(E, \nabla) \rightarrow\left(E^{\prime \prime}, \nabla^{\prime \prime}\right)$ is called exact if it is as sequence of bundle maps. Simpson calls $(E, \nabla)$ a regular singular $D_{U^{-m o d u l e, ~[21] . ~}}$.

If $v \in \Omega^{0}(E)$ and $f \in \Omega^{0}$ then

$$
\nabla(f v)=\left(\frac{\mathrm{d} f}{\mathrm{~d} z}\right) z v \frac{\mathrm{d} z}{z}+f \nabla(v) \quad \text { so } \quad(\nabla(f v))(0)=f(0)(\nabla(v))(0) .
$$

Hence, $\nabla$ induces a canonical endomorphism

$$
\rho^{\prime}: E_{0} \rightarrow E_{0} \otimes\left(\mathcal{K}_{U} \otimes[0]\right)_{0}
$$

Definition 2.1.2 The canonical map $\rho: E_{0} \rightarrow E_{0}$, determined by $\rho^{\prime}(w)=$ $\rho(w) \cdot(d z / z)(0)$ for all $w \in E_{0}$, is called the residue of $\nabla$ at 0 . If $\left(\lambda^{1}, \ldots, \lambda^{r}\right)$ denote the eigenvalues of $\rho$ and $\phi^{i}:=\left[-\operatorname{Re}\left(\lambda^{i}\right)\right] \in \mathbb{Z}$ then $\phi^{1} \geq \ldots \geq \phi^{r}$ are called the integer weights of $\nabla$ (at 0$)$.

We encode the integer weights as

$$
\Phi:=\operatorname{diag}\left(\phi^{i}\right)=\operatorname{block-\operatorname {diag}}\left(\psi^{m} I_{d^{m}}\right)
$$


with $\psi^{i}>\psi^{i+1}$ for all $i$. $\Phi$ induces a canonical block structure on all matrices which we will use a lot. If $\nabla_{0}$ is a second logarithmic connection on $E$, the Leibnitz rule implies that

$$
\left(\nabla_{0}-\nabla\right): E \rightarrow E \otimes \mathcal{K}_{U} \otimes[0]
$$

is a holomorphic bundle map. So, in a trivialisation $\theta: E \rightarrow U \times \mathbb{C}^{r}$,

$$
\nabla_{\theta}:=\theta \circ \nabla \circ \theta^{-1}=\mathrm{d}+A(z) \frac{\mathrm{d} z}{z}
$$

for holomorphic $A: U \rightarrow \operatorname{End}\left(\mathbb{C}^{r}\right)$. The converse clearly holds. Furthermore, $\rho=\theta(0)^{-1} \circ A(0) \circ \theta(0)$.

\subsection{Gauge fixing for logarithmic connections}

If all eigenvalues $\mu$ of $K \in \operatorname{End}\left(\mathbb{C}^{r}\right)$ satisfy $\operatorname{Re}(\mu) \in[0,1)$ then we say $K$ has normalised eigenvalues. If also $G=\exp (2 \pi i K)$ we call $K$ the normalised logarithm of $G$ : $K=$ norm $\log G$. If $G$ is upper-triangular and has only one eigenvalue $\rho$ then, for $\mu=\operatorname{norm} \log \rho$, we have, [15, p 376],

$$
\text { norm } \log G=\mu I+\frac{1}{2 \pi i} \sum_{j=1}^{\infty} \frac{(-1)^{j}}{j}\left(\frac{1}{\rho} G-I\right)^{j} .
$$

Definition 2.2.1 A trivialisation $\theta:(E, \nabla) \rightarrow\left(U \times \mathbb{C}^{r}, \nabla_{\theta}\right)$ is called normal (w.r.t. z) if

$$
\nabla_{\theta}=d+z^{\Phi}(-K-\Phi) z^{-\Phi} \frac{d z}{z}
$$

for some constant, block-upper-triangular $K \in \operatorname{End}\left(\mathbb{C}^{r}\right)$ with normalised eigenvalues, where $\Phi$ is the integer weights-matrix of $(E, \nabla)$.

For a normal trivialisation $\theta$, integer weights $\Phi=$ block-diag $\left(\psi^{m} I_{d^{m}}\right)$ and $\left(e_{1}, \ldots, e_{r}\right)$ the standard frame of $U \times \mathbb{C}^{r}$, let

$$
F^{m}:=\left\langle e_{d^{1}+\ldots+d^{m-1}+1}, \ldots, e_{d^{1}+\ldots+d^{m}}\right\rangle \subseteq U \times \mathbb{C}^{r}
$$

for $m=1, \ldots, l$ and $F^{0}:=U \times\{0\}$. Set

$$
\begin{aligned}
& \phi: U \times \mathbb{C}^{r} \rightarrow \mathbb{Z} \cup\{+\infty\} \\
& v \mapsto \begin{cases}\psi^{m} & \text { if } \quad v \in\left(\oplus_{0}^{m} F^{k}\right)-\left(\oplus_{0}^{m-1} F^{k}\right) \\
+\infty & \text { if } \quad v=0 .\end{cases}
\end{aligned}
$$

Clearly, $\phi$ is invariant under parallel transport away from the singularity. 
Remark 2.2.2 For $v \in U^{*} \times \mathbb{C}^{r}, \phi(v)$ can be described as the integer part

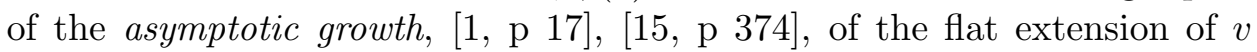
over $U^{*}$. This follows from Lemma 2.2.5 and equations (2.2.8), (2.2.11) and

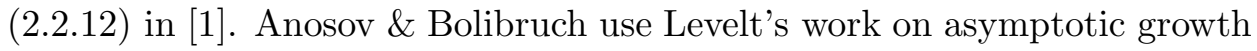
for regular systems, i.e. singular connections such that flat sections have at most polynomial growth. We avoid regular systems.

Under the equivalence of asymptotic growth and $\phi$, (i) and the first part of (iii) of the following theorem correspond to results of Levelt, [1, p 28], [4, p 60], [15, p 379]. Gantmacher has (i), [9, p 185,191].

Theorem 2.2.3 (i) For each logarithmic connection (and any coordinate), there exists a normal trivialisation in some small neighbourhood of the singularity.

(ii) Let $\tau:\left(E^{\prime}, \nabla^{\prime}\right) \rightarrow(E, \nabla)$ and consider two normal trivialisations $\theta$ : $(E, \nabla) \rightarrow\left(U \times \mathbb{C}^{r}, \nabla_{\theta}\right)$ w.r.t. $z$ and $\theta^{\prime}:\left(E^{\prime}, \nabla^{\prime}\right) \rightarrow\left(U \times \mathbb{C}^{r^{\prime}}, \nabla_{\theta^{\prime}}^{\prime}\right)$ w.r.t. $u$. Then $M:=\theta \circ \tau \circ\left(\theta^{\prime}\right)^{-1}$ satisfies

$$
\phi\left(M\left(v^{\prime}\right)\right) \geq \phi^{\prime}\left(v^{\prime}\right)
$$

for all $v^{\prime} \in U \times \mathbb{C}^{r^{\prime}}$.

(iii) If $\tau$ is injective then $\phi\left(M\left(v^{\prime}\right)\right)=\phi^{\prime}\left(v^{\prime}\right)$ for all $v^{\prime} \in U \times \mathbb{C}^{r^{\prime}}$. If $\tau$ is surjective and $v \in U \times \mathbb{C}^{r}$ then there exists $v^{\prime} \in \tau^{-1}(v)$ such that $\phi(v)=\phi^{\prime}\left(v^{\prime}\right)$.

We give a direct proof.

Proof: (i): Start with any trivialisation and write the connection as $\mathrm{d}+$ $A(z) d z / z$ for $A(z)=\sum_{0}^{\infty} A^{j} z^{j}$. Applying a constant gauge transformation, we can assume $A^{0}=$ block-diag $\left(A_{m, m}^{0}\right)$ where each eigenvalue $\lambda$ of $A_{m, m}^{0}$ satisfies

$$
[-\operatorname{Re} \lambda]=\psi^{m}, \quad \text { i.e. } \quad-\operatorname{Re} \lambda-\psi^{m} \in[0,1) .
$$

Assume we could find $M(z)=\sum_{0}^{\infty} M^{j} z^{j}: U \rightarrow \mathrm{Gl}\left(r, \mathbb{C}^{\prime}\right)$ and $B(z)=\sum_{0}^{\infty} B^{j} z^{j}: U \rightarrow \operatorname{End}\left(\mathbb{C}^{r}\right)$ such that

$$
M^{-1} \circ\left(\mathrm{d}+A(z) \frac{\mathrm{d} z}{z}\right) \circ M=\mathrm{d}+B(z) \frac{\mathrm{d} z}{z}
$$

with $M^{0}=I$, hence $B^{0}=A^{0}$, and $B(z)=z^{\Phi}(-K-\Phi) z^{-\Phi}$ for $K$ constant, block-upper-triangular. Then the eigenvalues of $K$ would be those of $-B^{0}-$ 
$\Phi=-A^{0}-\Phi$ and hence $K$ would have normalised eigenvalues by (2). We would be done if the series of $M$ converges in a small neighbourhood of 0 .

Eq. (3) is equivalent to

$$
\mathrm{d}+M^{-1}(\mathrm{~d} M)+M^{-1} A M \frac{\mathrm{d} z}{z}=\mathrm{d}+B \frac{\mathrm{d} z}{z}
$$

i.e. $z \mathrm{~d} M=(M B-A M) \mathrm{d} z$. In the Taylor expansion we must have

$$
j M^{j}=\sum_{k=0}^{j}\left\{M^{k} B^{j-k}-A^{j-k} M^{k}\right\} \quad \forall j \geq 0 .
$$

So (3) is fulfilled if

$$
\left(j M^{j}+A^{0} M^{j}-M^{j} B^{0}\right)-B^{j}=-A^{j}+\sum_{k=1}^{j-1}\left\{M^{k} B^{j-k}-A^{j-k} M^{k}\right\}=: R^{j-1}
$$

for all $j \geq 1$. Work by induction on $j \geq 1$. For all $i, m=1, \ldots, l$, we need to satisfy, for $M^{0}=I\left(A^{0}=B^{0}\right)$, the equation on the block entries

$$
\left(j+A_{i, i}^{0}\right) M_{i, m}^{j}-M_{i, m}^{j} A_{m, m}^{0}-B_{i, m}^{j}=R_{i, m}^{j-1} .
$$

By (2), any eigenvalue $\lambda^{\prime \prime}$ of $\left(j+A_{i, i}^{0}\right)$ satisfies $\left[-\operatorname{Re} \lambda^{\prime \prime}\right]=-j+\psi^{i}$. So, $\lambda^{\prime \prime}$ is not an eigenvalue of $A_{m, m}^{0}$ unless $\psi^{i}-j=\psi^{m}$. Hence there is a solution $\left(M_{i, m}^{j}, B_{i, m}^{j}\right)$ with $B_{i, m}^{j}=0$ if $\psi^{i}-j \neq \psi^{m}$. So we find $M$ and $B$ as required.

To see that $\sum_{0}^{\infty} M^{j} z^{j}$ is absolutely convergent near 0 , set

$$
c_{j}:=\left\|A^{j}\right\|+\left\|B^{j}\right\| \quad \forall j \geq 1 \quad \text { and } \quad c_{0}:=2\left[\left\|A^{0}\right\|\right]+2 \in \mathbb{Z}
$$

in operator norm. We can find $C>1$ and $\varepsilon_{0}>0$ such that

$$
c_{j} \varepsilon^{j}<C \quad \forall j \geq 0 \quad \forall 0 \leq \varepsilon \leq \varepsilon_{0}
$$

since $A$ and $B$ are absolutely convergent. The equality (4) implies

$$
\left(j-c_{0}\right)\left\|M^{j}\right\| \leq \sum_{k=0}^{j-1}\left\|M^{k}\right\| c_{j-k} .
$$

Choose any $\delta \leq \varepsilon_{0} / 2 C$. Then $(2 C \delta)^{j-k} c_{j-k} \leq C$ for all $j-k$ and so

$$
\left(j-c_{0}\right)\left\|M^{j}\right\|(2 C \delta)^{j} \leq C \sum_{k=0}^{j-1}\left\|M^{k}\right\|(2 C \delta)^{k} .
$$


Hence

$$
\left(j-c_{0}\right)\left\|M^{j}\right\| \delta^{j} \leq 2^{-j} \sum_{0}^{j-1}\left\|M^{k}\right\|(2 \delta)^{k} .
$$

Let $D:=\sum_{k=0}^{c_{0}}\left\|M^{k}\right\| \delta^{k}$. Then we claim that

$$
\left\|M^{j}\right\| \delta^{j} \leq D 2^{c_{0}-j} \quad \forall j \geq c_{0}
$$

which would finish (i). The claim is clear for $j=c_{0}$ and for $j>c_{0}$ we use induction to find

$$
\begin{aligned}
\left\|M^{j}\right\| \delta^{j} & \leq \frac{2^{-j}}{j-c_{0}}\left(\sum_{0}^{c_{0}}\left\|M^{k}\right\| \delta^{k} 2^{c_{0}}+\sum_{c_{0}+1}^{j-1} D 2^{c_{0}-k} 2^{k}\right) \\
& \leq 2^{c_{0}-j} D \frac{1+(j-1)-c_{0}}{j-c_{0}} .
\end{aligned}
$$

(ii): By hypothesis,

$$
\begin{gathered}
\nabla_{\theta^{\prime}}=\mathrm{d}+B(z) \frac{\mathrm{d} z}{z}=\mathrm{d}+u^{\Phi^{\prime}}\left(-K^{\prime}-\Phi^{\prime}\right) u^{-\Phi^{\prime}} \frac{\mathrm{d} u}{u} \\
\nabla_{\theta}=\mathrm{d}+A(z) \frac{\mathrm{d} z}{z}=\mathrm{d}+z^{\Phi}(-K-\Phi) z^{-\Phi} \frac{\mathrm{d} z}{z}
\end{gathered}
$$

and $M=\sum_{0}^{\infty} M^{j} z^{j}: U \rightarrow \operatorname{Hom}\left(\mathbb{C}^{r^{\prime}}, \mathbb{C}^{r}\right)$ with $M \circ \nabla_{\theta^{\prime}}^{\prime}=\nabla_{\theta} \circ M$, i.e. (四) above.

Let $M=\oplus_{i, m} M_{i, m}$ for $M_{i, m}: F^{\prime m} \rightarrow F^{i}$ where $\mathbb{C}^{r^{\prime}}=\oplus_{1}^{l^{\prime}} F^{\prime m}$ and $\mathbb{C}^{r}=$ $\oplus_{1}^{l} F^{i}$ according to $\Phi^{\prime}$ and $\Phi$, respectively. If $M$ is block-upper-triangular, i.e. $M_{i, m}=0$ if $\psi^{\prime m}>\psi^{i}$, then (ii) follows.

For $j=0$, (41) gives $A^{0} M^{0}-M^{0} B^{0}=0$. Clearly, $B^{0}$ and $A^{0}$ are blockdiagonal, any eigenvalue $\lambda^{\prime}$ of $B_{m, m}^{0}$ satisfies $\left[-\operatorname{Re} \lambda^{\prime}\right]=\psi^{\prime m}$ and any eigenvalue $\lambda$ of $A_{i, i}^{0}$ satisfies $[-\operatorname{Re} \lambda]=\psi^{i}$. This implies

$$
M_{i, m}^{0}=0 \quad \text { if } \quad \psi^{\prime m} \neq \psi^{i} .
$$

Since $A$ and $B$ are block-upper-triangular, (ब) implies by induction on $j \geq 0$ that $j M^{j}+A^{0} M^{j}-M^{j} B^{0}$ is block-upper-triangular, i.e.

$$
\left(j+A_{i, i}^{0}\right) M_{i, m}^{j}-M_{i, m}^{j} B_{m, m}^{0}=0
$$

if $\psi^{\prime m}>\psi^{i}>\psi^{i}-j$. Hence, $M^{j}$ is block-upper-triangular. 
(iii): Assume $\tau$ is injective and $v^{\prime} \in U \times \mathbb{C}^{r^{\prime}}$ has $\phi^{\prime}\left(v^{\prime}\right)=\psi^{\prime m}$. By (5), there exists $i$ such that $\psi^{i}=\psi^{\prime m}$ and $M_{i, m}^{0}$ has full rank. Since $\phi$ and $\phi^{\prime}$ are invariant under parallel transport, $v^{\prime}$ is, w.l.o.g., contained in the neighbourhood of 0 where $M_{i, m}$ has full rank. Hence, $M\left(v^{\prime}\right)$ has a component in $F^{i}$ and so, $\phi\left(M\left(v^{\prime}\right)\right) \leq \psi^{i}$.

Now assume $\tau$ is surjective and $v \in U \times \mathbb{C}^{r}$ with $\phi(v)=\psi^{i}$. Write $v=v^{1}+\ldots+v^{i}$ according to the decomposition of $\mathbb{C}^{r}$. By the dual of the above argument, we find $v^{\prime}=v^{\prime 1}+\ldots+v^{\prime i}$ with $v^{k}=M\left(v^{\prime k}\right)$ and $\phi^{\prime}\left(v^{\prime k}\right)=\psi^{k}$. Hence, $\phi^{\prime}\left(v^{\prime}\right)=\psi^{i}$.

Definition 2.2.4 Let $\theta$ be a normal trivialisation of $(E, \nabla)$. The integer weights filtration of $(E, \nabla)$ is

$$
0 \subset E^{1} \subset \ldots \subset E^{l}=E \quad \text { where } \quad E^{m}:=\theta^{-1}\left(\oplus_{1}^{m} F^{k}\right) .
$$

At first, the normal trivialisation $\theta$ and hence the filtration of $E$ exists only over a small neighbourhood of the singularity. But since each $E^{m}$ is invariant under $\nabla$, we can extend over all of $U$. This filtration, together with $\Phi$, is equivalent to $\phi \circ \theta: E \rightarrow \mathbb{Z} \cup\{+\infty\}$. It is independent of the choice of $\theta$ by Theorem 2.2.3.

Let $\pi: \tilde{U}^{*} \rightarrow U^{*}$ be the universal covering and write $\tilde{z}$ for the coordinate over $z$. Let $\log \tilde{z}: \tilde{U}^{*} \rightarrow \mathscr{C}$ be a holomorphic function such that $\log \tilde{z} \equiv$ $\log z \bmod (2 \pi i)$. For $K \in \operatorname{End}\left(\mathbb{C}^{r}\right)$ let $\tilde{z}^{K}:=\exp (K \log \tilde{z}) ; \tilde{z}^{\Phi}=z^{\Phi}$.

Lemma 2.2.5 For $\theta$ as in Definition 2.2.1, $\nabla_{\theta}\left(z^{\Phi} \tilde{z}^{K}\right)=0$ on $\tilde{U}^{*}$, i.e. $z^{\Phi} \tilde{z}^{K}$ is a fundamental system of flat sections. Hence, $\exp (2 \pi i K)$ is the monodromy around 0 .

Proof: $\left(\mathrm{d}+z^{\Phi}(-K-\Phi) z^{-\Phi} \frac{d z}{z}\right)\left(z^{\Phi} \tilde{z}^{K}\right)=z^{\Phi}(\Phi+K-K-\Phi) \tilde{z}^{K} \frac{d z}{z}$.

\subsection{Correspondence between local logarithmic connections and weighted flat bundles}

Definition 2.3.1 (Simpson, Deligne) A weighted flat bundle $\left(H, H^{m}, \Phi\right)$ over $U^{*}$ consists of a holomorphic, rank $r$ vector bundle $H \rightarrow U^{*}$ with a holomorphic (i.e. flat and compatible) connection

$$
\nabla: \Omega^{0}(H) \rightarrow \Omega^{0}(H) \otimes \Omega_{U^{*}}^{1}
$$


a filtration by proper subbundles $0 \subset H^{1} \subset \ldots \subset H^{l}=H$, invariant under $\nabla$, and an $r \times r$ matrix with integer entries $\Phi=\operatorname{diag}\left(\phi^{i}\right)=$ block-diag $\left(\psi^{m} I_{d^{m}}\right)$ where $\psi^{m}>\psi^{m+1}$ and $d^{m}=\operatorname{rank}\left(H^{m} / H^{m-1}\right)$.

The integer $\psi^{m}$ is called the integer weight of $H^{m}$. The function

$$
\begin{aligned}
& \phi: H \rightarrow \mathbb{Z} \cup\{+\infty\} \\
& v \mapsto \begin{cases}\psi^{m} & \text { if } \quad v \in H^{m} \backslash H^{m-1} \\
+\infty & \text { if } \quad v=0\end{cases}
\end{aligned}
$$

is equivalent to the filtration and weights; $(H, \phi):=\left(H, H^{m}, \Phi\right)$. A morphism of weighted flat bundles $\eta:\left(H^{\prime}, \phi^{\prime}\right) \rightarrow(H, \phi)$ is a map of flat bundles such that

$$
\phi\left(\eta\left(v^{\prime}\right)\right) \geq \phi^{\prime}\left(v^{\prime}\right)
$$

for all $v^{\prime} \in H^{\prime}$. Equivalently, $\eta\left(H^{\prime k}\right) \subseteq H^{m-1}$ if $\psi^{\prime k}>\psi^{m}$. The morphism is called injective if it is as bundle map and satisfies $\phi\left(\eta\left(v^{\prime}\right)\right)=\phi^{\prime}\left(v^{\prime}\right)$ for all $v^{\prime} \in$ $H^{\prime}$. It is called surjective if it is as bundle map and if for all $v \in H$ there exists $v^{\prime} \in \eta^{-1}(v)$ such that $\phi(v)=\phi^{\prime}\left(v^{\prime}\right)$. A sequence $\left(H^{\prime}, \phi^{\prime}\right) \stackrel{\eta}{\rightarrow}(H, \phi) \stackrel{\xi}{\rightarrow}\left(H^{\prime \prime}, \phi\right)$ is called exact if $\eta$ is injective, $\xi$ is surjective and $\operatorname{Im} \eta=\operatorname{Ker} \xi$. The direct $\operatorname{sum}\left(H^{\prime}, \phi^{\prime}\right) \oplus\left(H^{\prime \prime}, \phi^{\prime \prime}\right)$ is defined by $\left(H^{\prime} \oplus H^{\prime \prime}, \phi\right)$ where $\phi\left(h^{\prime} \oplus h^{\prime \prime}\right):=$ $\min \left(\phi^{\prime}\left(h^{\prime}\right), \phi^{\prime \prime}\left(h^{\prime \prime}\right)\right)$.

Definition 2.3.2 Let $\mathcal{F}^{-1}$ be the functor from the category of logarithmic connections to the category of weighted flat bundles, given by restricting the integer weights filtration and the morphisms to $U^{*}$.

By Theorem 2.2.3, $\mathcal{F}^{-1}$ sends injections, surjections and short exact sequences to such. To construct a functor $\mathcal{F}$, inverse to $\mathcal{F}^{-1}$, consider a weighted flat bundle $(H, \phi)$. Let $Y=\left(y_{1}, \ldots, y_{r}\right)$ be a fundamental system of multivalued flat sections, such that $\left\langle y_{1}, \ldots, y_{d^{1}+\ldots+d^{m}}\right\rangle=H^{m}$.

Let $\gamma$ be a loop in $U^{*}$ going once around 0 anticlockwise and write $\gamma^{*}$ for the induced action on $\tilde{U}^{*} ; \log \left(\tilde{z} \circ \gamma^{*}\right)=(\log \tilde{z})+2 \pi i$. Then

$$
Y \circ \gamma^{*}=Y G
$$

for constant block-upper-triangular $G \in \operatorname{Gl}(r, \mathscr{C})$. Put $K:=\operatorname{norm} \log G$ which is also block-upper-triangular. Since $\tilde{z}^{-K} z^{-\Phi}$ is invertible over $\tilde{U}^{*}$, $Y \tilde{z}^{-K} z^{-\Phi}$ is a trivialisation of $H$ over $\tilde{U}^{*}$. It is single valued, [1, p 17], since

$$
\left(Y \tilde{z}^{-K} z^{-\Phi}\right) \circ \gamma^{*}=Y G G^{-1} \tilde{z}^{-K} z^{-\Phi} .
$$


Definition 2.3.3 Let $\mathcal{F}(H, \phi)$ be the extension of $H$ over $U$, whose stalk at 0 is generated by the system of sections

$$
V(z):=Y \tilde{z}^{-K} z^{-\Phi}: U^{*} \rightarrow H \times \cdots \times H .
$$

$\nabla$ becomes a singular connection on the extension of $H$. For a morphism $\eta$ : $\left(H^{\prime}, \phi^{\prime}\right) \rightarrow(H, \phi)$, let $\mathcal{F}(\eta): \mathcal{F}\left(H^{\prime}, \phi^{\prime}\right) \rightarrow \mathcal{F}(H, \phi)$ be the unique holomorphic extension of $\eta$.

$\mathcal{F}(H, 0)$ is called the canonical extension, [17], of the flat bundle $H$. One checks that different choices of $Y$ and coordinate $z$ give extensions which are isomorphic via a map extending the identity of $H$. Anosov \& Bolibruch construct extensions of $H$ by choosing $Y$ such that $G$ is upper-triangular, but with $Y$ not requested to respect a fixed filtration. One can (in addition) choose $Y$ such that $G$ decomposes w.r.t. eigenvalues. Assuming then that $G$ has only one eigenvalue, it is easy to see that $\mathcal{F}$ equals the extension-functor of Manin, [6, p 94], and is a special case of Simpson's extension functor, [21, $\mathrm{p}$ 738]. They have the following lemma.

Lemma 2.3.4 $\mathcal{F}(H, \phi)$ is a logarithmic connection and $\mathcal{F}$ is inverse to $\mathcal{F}^{-1}$ on objects.

$$
\begin{aligned}
& \text { Proof: } \quad \nabla(V(z))= \\
& Y \mathrm{~d}\left(\tilde{z}^{-K} z^{-\Phi}\right)=Y \tilde{z}^{-K}(-K-\Phi) z^{-\Phi} \frac{\mathrm{d} z}{z}=V(z) z^{\Phi}(-K-\Phi) z^{-\Phi} \frac{\mathrm{d} z}{z} .
\end{aligned}
$$

So, $\nabla_{V}=\mathrm{d}+z^{\Phi}(-K-\Phi) z^{-\Phi} \frac{d z}{z}$ in the trivialisation given by the columns of $V$. Combine this with Lemma 2.2.5.

Lemma 2.3.5 Let $G, G^{\prime} \in G l(r, \mathbb{C})$ and put $K:=\operatorname{norm} \log G, K^{\prime}:=$ norm $\log G^{\prime}$. If $C$ is an $r \times r^{\prime}$-matrix such that $G C=C G^{\prime}$ then $K C=C K^{\prime}$ and hence $\tilde{z}^{K} C=C \tilde{z}^{K^{\prime}}$. If $G G^{\prime}=G^{\prime} G$ then $K K^{\prime}=K^{\prime} K$.

Lemma 2.3.6 (Simpson, Deligne) The holomorphic extension $\mathcal{F}(\eta)$ in Definition 2.3.3 exists. It commutes with the logarithmic connections. If $\eta$ is injective (surjective) then so is $\mathcal{F}(\eta) . \mathcal{F}$ sends short exact sequences to such and is inverse to $\mathcal{F}^{-1}$ on morphisms. 
Proof: Since $\eta$ maps flat sections to flat sections we can find a unique constant $r \times r^{\prime}$-matrix $C$ such that $\eta \circ Y^{\prime}=Y C$. Because $\eta$ does not decrease weights, $z^{\Phi} C z^{-\Phi^{\prime}}$ is holomorphic over $U$. Furthermore,

$$
\left(\eta \circ Y^{\prime}\right) \circ \gamma^{*}=(Y C) \circ \gamma^{*} \text { so that }\left(\eta \circ Y^{\prime}\right) G^{\prime}=Y G C,
$$

i.e. $C G^{\prime}=G C$. By Lemma 2.3.5 this implies $\tilde{z}^{K} C=C \tilde{z}^{K^{\prime}}$. We find

$$
\mathcal{F}(\eta)\left(V^{\prime}\right)=\left(\eta \circ Y^{\prime}\right) \tilde{z}^{-K^{\prime}} z^{-\Phi^{\prime}}=V\left(z^{\Phi} \tilde{z}^{K} C \tilde{z}^{-K^{\prime}} z^{-\Phi^{\prime}}\right)=V\left(z^{\Phi} C z^{-\Phi^{\prime}}\right)
$$

and $\mathcal{F}(\eta)$ is holomorphic. If $\eta$ is injective (surjective) then we can choose $Y\left(Y^{\prime}\right)$ such that $C$ is a permutation matrix of full rank and $z^{\Phi} C z^{-\Phi^{\prime}}=C$. The remainder of the statement follows from continuity.

\section{Global logarithmic connections and weighted flat bundles}

\subsection{Correspondence over $\mathbb{P}^{1}$}

We extend the concepts to the Riemann sphere. Let $X=\left\{x_{1}, \ldots, x_{n}\right\} \subseteq \mathbb{P}^{1}$, put $S:=\mathbb{P}^{1}-X$ and fix a base point $s \in S$. For each $j=1, \ldots, n$ choose a simply connected neighbourhood $U_{j} \subseteq \mathbb{P}^{1}$ of $x_{j}$ containing $s$ but no other $x_{k}$ 's and a coordinate $z_{j}$ centered at $x_{j}$. ( $U_{j}$ is easier to handle than a small neighbourhood around $x_{j}$ and a path from $x_{j}$ to $s$.) Let $\gamma_{j} \in \pi_{1}\left(U_{j}^{*}, s\right)$ go once around $x_{j}$, anticlockwise, and $\tilde{U}_{j}^{*}$ be the universal covering of $U_{j}^{*}$.

A logarithmic connection over $\left(\mathbb{P}^{1}, X\right)$ consists of a holomorphic bundle $E \rightarrow \mathbb{P}^{1}$ and a $\mathbb{C}$-linear map $\nabla: \Omega^{0}(E) \rightarrow \Omega^{0}(E) \otimes \Omega^{1}(\log X)$ satisfying the Leibnitz rule; where $\Omega^{1}(\log X)=\Omega^{0}\left(\mathcal{K}_{\mathbb{P}^{1}} \otimes[X]\right)$.

A weighted flat bundle over $S$ is a holomorphic flat bundle $H \rightarrow S$ together with filtrations by flat subbundles $0 \subset H_{j}^{1} \subset \ldots \subset H_{j}^{l_{j}}=\left.H\right|_{U_{j}^{*}}$ and integer weights $\Phi_{j}=\operatorname{diag}\left(\phi_{j}^{i}\right)=\operatorname{block}-\operatorname{diag}\left(\psi_{j}^{m} I_{d_{j}^{m}}\right)$ for each $j=1, \ldots, n$. Note that no compatibility is required for different $j$. Write $\phi=\left(\phi_{1}, \ldots, \phi_{n}\right)$ for the weight functions $\phi_{j}:\left.H\right|_{U_{j}^{*}} \rightarrow \mathbb{Z} \cup\{+\infty\}$.

We have $\pi_{1}(S, s)=\left\langle\gamma_{1}, \ldots, \gamma_{n} \mid \gamma_{1} \cdot \ldots \cdot \gamma_{n}=1\right\rangle$ where $\gamma_{1} \cdot \gamma_{2}$ means travelling along $\gamma_{1}$ first. A weighted flat bundle corresponds to a conjugacy class of representations $\chi: \pi_{1}(S, s) \rightarrow \operatorname{Gl}(r, \mathbb{C})$ with, for each $j=1, \ldots, n$, a weighted filtration of $\mathbb{C}^{r}$ invariant under $\chi\left(\gamma_{j}\right)$. 
Corollary 3.1.1 ([21]) $\mathcal{F}$ induces an equivalence between the category of logarithmic connections over $\mathbb{P}^{1}$ and that of weighted flat bundles over $S$. It and its inverse preserve injections, surjections and short exact sequences.

Definition 3.1.2 (i) $\operatorname{deg}(H, \phi):=\sum_{j}\left\{\operatorname{Tr} \Phi_{j}+\operatorname{Tr}\left(\right.\right.$ norm $\left.\left.\log \chi\left(\gamma_{j}\right)\right)\right\} \in \mathbb{Z}$

(ii) A system (in one of the considered categories) of rank $r$ and degree $d$ is called stable if any proper subsystem of rank $r^{\prime}$ and degree $d^{\prime}$ satisfies $d^{\prime} / r^{\prime}<d / r$. For semi-stability allow ' $\leq$ '. The number $d / r$ is called the slope of the system.

Observe that $\operatorname{deg}(H, \phi)=\sum\left\{\operatorname{Tr} \Phi_{j}+\operatorname{Re} \operatorname{Tr}\left(\right.\right.$ norm $\left.\left.\log \chi\left(\gamma_{j}\right)\right)\right\}$ and it is an integer because $\operatorname{det} \chi\left(\gamma_{1}\right) \cdot \ldots \cdot \operatorname{det} \chi\left(\gamma_{n}\right)=1$.

Consider a weighted flat bundle $(H, \phi)$. Choose a basis $Y_{s}$ of $H_{s}$ and denote its extensions by parallel transport over $\tilde{U}_{j}^{*}$ by $Y\left(\tilde{z}_{j}\right)$. For each $j$, fix some $Z_{j} \in \operatorname{Gl}(r, \mathscr{C})$ such that $Y_{j}\left(\tilde{z}_{j}\right):=Y\left(\tilde{z}_{j}\right) Z_{j}$ respects the filtration of $\left.H\right|_{U_{j}^{*}}$. Let

$$
Y_{j}\left(\tilde{z}_{j}\right) \circ \gamma_{j}^{*}=Y_{j}\left(\tilde{z}_{j}\right) G_{j},
$$

$K_{j}:=\operatorname{norm} \log G_{j}$. Set $(E, \nabla)=\mathcal{F}(H, \phi)$. By the Birkhoff-GrothendieckTheorem (BGT), 18], [1], there is a system

$$
W: \mathbb{P}^{1} \rightarrow E \times \ldots \times E
$$

of $r$ meromorphic sections such that $\left.W\right|_{S}$ spans $H=\left.E\right|_{S}$. We have

$$
V_{j}\left(z_{j}\right):=Y_{j}\left(\tilde{z}_{j}\right) \tilde{z}_{j}^{-K_{j}} z_{j}^{-\Phi_{j}}=W\left(z_{j}\right) Q_{j}\left(z_{j}\right),
$$

for some meromorphic $Q_{j}: U_{j} \rightarrow \mathrm{Gl}(r, \mathbb{C})$, holomorphic on $U_{j}^{*}$. Note that $\left.W\right|_{U_{j}}$ spans $\left.E\right|_{U_{j}}$ if and only if $Q_{j}$ is holomorphic at $x_{j}$.

Proposition 3.1.3 ([15, p 32], [21, p 754]) $\mathcal{F}$ preserves degrees and so (semi-) stability.

Proof: Let $\omega$ be the connection matrix of $\left.\nabla\right|_{H}$ w.r.t. the trivialisation $\left.W\right|_{H}$. $\operatorname{Tr} \omega$ is a single valued holomorphic one-form on $S$. By the Residue-Theorem, $0=\sum_{1}^{n} \operatorname{Res}_{x_{j}}(\operatorname{Tr} \omega)$. Since $\nabla\left(\left.W\right|_{U_{j}^{*}}\right)=Y_{j}\left(\tilde{z}_{j}\right) \mathrm{d}\left(\tilde{z}_{j}^{-K_{j}} z_{j}^{-\Phi_{j}} Q_{j}\left(z_{j}\right)^{-1}\right)$,

$$
\begin{aligned}
\left.\omega\right|_{U_{j}^{*}} & =Q_{j}\left(z_{j}\right) z_{j}^{\Phi_{j}} \tilde{z}_{j}^{K_{j}} \mathrm{~d}\left(\tilde{z}_{j}^{-K_{j}} z_{j}^{-\Phi_{j}} Q_{j}\left(z_{j}\right)^{-1}\right) \\
& =-Q_{j}\left[z_{j}^{\Phi_{j}} K_{j} z_{j}^{-\Phi_{j}}+\Phi_{j}\right] Q_{j}^{-1} \frac{\mathrm{d} z_{j}}{z_{j}}+Q_{j} \mathrm{~d}\left(Q_{j}^{-1}\right) .
\end{aligned}
$$


Let $k_{j}$ be the order of vanishing of $\operatorname{det} Q_{j}$ at $x_{j}$. Then

$$
\left.\operatorname{Tr} \omega\right|_{U_{j}^{*}}=-\left(\operatorname{Tr} K_{j}+\operatorname{Tr} \Phi_{j}+k_{j}\right) \frac{\mathrm{d} z_{j}}{z_{j}}+\alpha
$$

for a holomorphic 1-form $\alpha$. Hence $-\sum_{j} k_{j}=\sum_{j} \operatorname{Tr} K_{j}+\operatorname{Tr} \Phi_{j}$. The righthand-side is the degree of the weighted flat bundle, while the left-hand-side is the sum of the orders of vanishing of $\operatorname{det} W$, the degree of $E$.

This result holds, in fact, over any Riemann surface. Note, that it implies Lemma 5.2.2 in [1]. Also, if $(E, \nabla) \rightarrow \mathbb{P}^{1}$ is logarithmic at $X$ and has residues $\rho_{j}: E_{x_{j}} \rightarrow E_{x_{j}}$ then $-\sum_{1}^{n} \operatorname{Tr} \rho_{j}$ equals the degree of $E$. This is because in a normal trivialisation, using the notation of Definition 2.2.1, $\rho_{j}=\left(z_{j}^{\Phi_{j}}\left(-K_{j}-\Phi_{j}\right) z_{j}^{-\Phi_{j}}\right)(0)$. Hence, $-\sum \operatorname{Tr} \rho_{j}=\sum\left(\operatorname{Tr} K_{j}+\operatorname{Tr} \Phi_{j}\right)=$ $\operatorname{deg} \mathcal{F}^{-1}(E, \nabla)$.

\subsection{The splitting type of $E \rightarrow \mathbb{P}^{1}$}

By the BGT, any holomorphic bundle $E \rightarrow \mathbb{P}^{1}$ has the form $E \cong \mathcal{O}\left(c_{1}\right) \oplus$ $\ldots \oplus \mathcal{O}\left(c_{r}\right)$ for unique integers $c_{1} \geq \ldots \geq c_{r}$. We call $C:=\operatorname{diag}\left(c_{i}\right)$ the splitting type of $E$ or $(E, \nabla)$. If $C=c_{1} I_{r}$ we say $E$ has constant splitting type. Recall that $\mathrm{H}^{0}(\mathcal{O}(c))$ is zero if $c<0$ and equal to $\mathbb{C}$ if $c=0$. As $\mathcal{F}$ preserves subsystems and degrees, this implies Theorem 5.2.2 in [1].

Theorem 3.2.1 If $(E, \nabla)$ is semi-stable, $n \geq 2$ and $C=\operatorname{diag}\left(c_{i}\right)$ the splitting type, then $(0 \leq) c_{i}-c_{i+1} \leq n-2$ for all $i=1, \ldots, r-1$.

Proof: Fix a splitting $E=\mathcal{O}\left(c_{1}\right) \oplus \ldots \oplus \mathcal{O}\left(c_{r}\right)$. Suppose there exists an $i \in\{1, \ldots, r-1\}$ such that $c_{i}-c_{i+1}>n-2$. Let $z$ be a coordinate centred at $s \in S$. For all $k \in\{1, \ldots, i\}$ we can find sections $v_{k}$ of $\mathcal{O}\left(c_{k}\right)$ vanishing to order $c_{k}$ at $s$, i.e. $v_{k} z^{-c_{k}}$ spans $\mathcal{O}\left(c_{k}\right)$ near $s$. For each $m \in\{i+1, \ldots, r\}$ we consider the natural projection

$$
\pi_{m}: E \otimes \mathcal{K}_{\mathbb{P}^{1}} \otimes[X] \rightarrow \mathcal{O}\left(c_{m}\right) \otimes \mathcal{K}_{\mathbb{P}^{1}} \otimes[X]
$$

and obtain sections $\pi_{m} \circ \nabla\left(v_{k}\right): \mathbb{P}^{1} \rightarrow \mathcal{O}\left(c_{m}\right) \otimes \mathcal{K}_{\mathbb{P}^{1}} \otimes[X]$. Near $s$ we have

$$
\begin{aligned}
\pi_{m} \circ \nabla\left(v_{k}\right) & =\pi_{m} \circ \nabla\left(v_{k} z^{-c_{k}} z^{c_{k}}\right) \\
& =\pi_{m}\left(\nabla\left(v_{k} z^{-c_{k}}\right) z^{c_{k}}+\left(v_{k} z^{-c_{k}}\right) \mathrm{d}\left(z^{c_{k}}\right)\right) \\
& =\pi_{m}\left(\nabla\left(v_{k} z^{-c_{k}}\right) z^{c_{k}}\right)
\end{aligned}
$$


since $k \neq m$. Either $\pi_{m} \circ \nabla\left(v_{k}\right)$ is identically zero or of order at least $c_{k}$. The latter is equivalent to

$$
c_{k} \leq \operatorname{deg}\left(\mathcal{O}\left(c_{m}\right) \otimes \mathcal{K}_{\mathbb{P}^{1}} \otimes[X]\right)=c_{m}+n-2 .
$$

But from $k<i<m$ we see that $c_{k} \geq c_{i}>c_{i+1}+n-2 \geq c_{m}+n-2$. Therefore, $\pi_{m} \circ \nabla\left(v_{k}\right)$ is identically zero for all $k<i<m$. So, $\nabla$ preserves $\mathcal{O}\left(c_{1}\right) \oplus \ldots \oplus \mathcal{O}\left(c_{i}\right)$. Semistability implies then that $c_{1}=c_{2}=\ldots=c_{r}$.

This theorem easily extends to logarithmic connections with parabolic structure at the singularities, i.e. to filtered regular $D_{S}$-modules, c.f. [21]. Note, any logarithmic connection with irreducible monodromy is semi-stable, even stable.

Corollary 3.2.2 (Bolibruch, [1, p 84]) If the monodromy of $(E, \nabla)$ is irreducible $(n \geq 2)$ then $\sum_{i=1}^{r} c_{1}-c_{i} \leq(n-2) r(r-1) / 2$.

Lemma 3.2.3 If $\mathcal{F}\left(H, H_{j}^{m}, \Phi_{j}\right)$ has splitting type $C$ and $\Phi_{j}^{\prime}=\Phi_{j}+\lambda_{j} I_{r}$ then $\mathcal{F}\left(H, H_{j}^{m}, \Phi_{j}^{\prime}\right)$ has splitting type $C+\left(\sum_{1}^{n} \lambda_{j}\right) I_{r}$.

Lemma 3.2.4 Let $\left(H^{\prime}, \phi^{\prime}\right) \rightarrow(H, \phi) \rightarrow\left(H^{\prime \prime}, \phi^{\prime \prime}\right)$ be a short exact sequence and assume that two of them have the same slope. Then all three have the same slope and $\mathcal{F}(H, \phi)$ has constant splitting type $C=c I_{r}$ if and only if $\mathcal{F}\left(H^{\prime}, \phi^{\prime}\right)$ and $\mathcal{F}\left(H^{\prime \prime}, \phi^{\prime \prime}\right)$ have constant splitting types $C^{\prime}=c I_{r^{\prime}}$ and $C^{\prime \prime}=$ $c I_{r^{\prime \prime}}$, respectively.

The proofs of these two lemmas are straightforward.

\section{The Riemann-Hilbert-Problem}

\subsection{Commutative and semi-simple representations}

Lemma 4.1.1 (泪, [1, p 76]) If $\chi: \pi_{1}(S) \rightarrow G l(r, \mathscr{C})$ factors through $H_{1}(S)$ then it is the monodromy of a Fuchsian system.

Proof: Since the $G_{j}=\chi\left(\gamma_{j}\right)$ commute, each $G_{j}$ preserves $\operatorname{ker}\left(G_{k}-\mu I\right)^{t}$ of each $G_{k}$ and for each $t$. Assume then that each $G_{j}$ has only one eigenvalue $\rho_{j}$ and is upper-triangular. Let $\mu_{j}:=$ norm $\log \rho_{j}$ be the only eigenvalue of $K_{j}:=\operatorname{norm} \log G_{j}$ and $\xi:=\sum_{1}^{n} \mu_{j} \in \mathbb{Z}$. By Lemma 2.3.5 the $K_{j}$ 's 
commute and $\exp \left(\xi \cdot I_{r}-\sum K_{j}\right)=G_{1} \cdot \ldots \cdot G_{n}=I_{r}$. Since $\xi \cdot I_{r}-\sum K_{j}$ has only the eigenvalue 0 , it is the normalised logarithm of $I_{r}$, i.e. 0 . A short calculation then shows that

$$
\nabla:=\mathrm{d}+\left[\frac{\xi}{z-x_{1}}-\sum_{j=1}^{n} \frac{K_{j}}{z-x_{j}}\right] \mathrm{d} z
$$

is smooth at infinity. Over each $\tilde{U}_{k}^{*}$ we set $Y:=\left(z-x_{1}\right)^{-\xi} \prod_{1}^{n}\left(z \widetilde{-} x_{j}\right)^{K_{j}}$ and find $\nabla(Y)=0$ and $Y \circ \gamma_{j}=Y G_{j}$.

Proposition 4.1.2 ([1, $\mathbf{p}$ 80]) If $(E, \nabla)=\mathcal{F}(H, \phi)$ has splitting type $C$ and $k \in\{1, \ldots, n\}$ is fixed then there exists a permutation $P$ and meromorphic $W: \mathbb{P}^{1} \rightarrow E \times \ldots \times E$ such that

(i) $W$ is holomorphic except at $x_{k}$ and spans $E$ away from $x_{k}$,

(ii) $W z_{k}^{-C}$ spans $E$ near $x_{k}$ and

(iii) $Q_{k}\left(z_{k}\right)=\hat{Q}_{k}\left(z_{k}\right) z_{k}^{-C} P=\hat{Q}_{k}\left(z_{k}\right) P z_{k}^{-P^{-1} C P}$ for some invertible $\hat{Q}_{k}$.

We give a proof different from that in [1].

Proof: Let $W^{\prime}=\left(w_{1}^{\prime}, \ldots, w_{r}^{\prime}\right)$ where $w_{i}^{\prime}: \mathbb{P}^{1} \rightarrow \mathcal{O}\left(c_{i}\right)$ vanishes to order $c_{i}$ at $s$ and the $\mathcal{O}\left(c_{i}\right)^{\prime}$ 's decompose $E$. Define $Q_{k}^{\prime}$ by $V_{k}=W^{\prime} Q_{k}^{\prime}$ near $x_{k}$, $\operatorname{det} Q_{k} \neq 0$.

Claim: For each permutation $P$ such that all bottom-right minors of $Q_{k}^{\prime}(0) P^{-1}$ are non-singular, there exists a $W$ as in the proposition. (The existence of such $P$ follows by induction from the description of the determinant of a matrix in terms of co-rank one minors.)

We may assume that $z_{k}(s)=\infty$. Suppose there exists $b=\left(\left(b_{i, j}\right)\right)$ : $\mathbb{P}^{1} \rightarrow \mathrm{Gl}(r, \mathscr{C})$ such that

$$
\begin{gathered}
b_{i, j}=\left\{\begin{array}{cc}
\sum_{0}^{c_{i}-c_{j}-1} b_{i, j}^{p} z_{k}^{p} & i<j \\
1 & i=j \\
0 & i>j,
\end{array}\right. \\
z_{k}^{c_{i}-c_{m}} \mid\left(b Q_{k}^{\prime} P^{-1}\right)_{i, m} \quad \forall i<m .
\end{gathered}
$$

Then $W=W^{\prime} b z_{k}^{C}$ spans $\left.E\right|_{\mathbb{P}^{1}-\left\{x_{k}\right\}}, W z_{k}^{-C}$ spans $E$ near $x_{k}$ and $W^{\prime} Q_{k}^{\prime}=$ $V_{k}=W Q_{k}$ implies

$$
Q_{k}=z_{k}^{-C} b Q_{k}^{\prime}=z_{k}^{-C}\left(b Q_{k}^{\prime} P^{-1}\right) P=\hat{Q}_{k} z_{k}^{-C} P
$$


for some invertible $\hat{Q}_{k}$ and we would be done. To find $b$ as in (6) satisfying (17) we need to solve a system of linear equations. With $Q_{k}^{\prime} P^{-1}=\left(\left(q_{j, m}\right)\right)$, condition $(\overline{7})$ is equivalent to

$$
z_{k}^{c_{i}-c_{m}} \mid \sum_{j=i+1}^{r} b_{i, j} q_{j, m}+q_{i, m}
$$

for all $1 \leq i<m \leq r$. Writing $q_{j, m}=\sum_{0}^{\infty} q_{j, m}^{p} z_{k}^{p}$, (7) becomes

$$
\sum_{j=i+1}^{r} \sum_{t=0}^{c_{i}-c_{j}-1} b_{i, j}^{t} q_{j, m}^{p-t}+q_{i, m}^{p}=0
$$

for all $1 \leq i<m \leq r$ and $0 \leq p<c_{i}-c_{m}$. We define

$$
\alpha(t):=\min \left\{j \in\{i+1, \ldots, r\} \mid t \leq c_{i}-c_{j}-1\right\} .
$$

Then (7) is equal to

$$
\sum_{t=0}^{p} \sum_{j=\alpha(t)}^{r} b_{i, j}^{t} q_{j, m}^{p-t}+q_{i, m}^{p}=0
$$

for all $1 \leq i<m \leq r$ and $0 \leq p<c_{i}-c_{m}$. Note that $\alpha(p) \leq m$. To find the $b_{i, j}^{t}$ 's we argue one row of $b$ at a time, i.e. fix $i$. Assume that $b_{i, j}^{t}$ is known by induction for all $t<p$. Then we have to fullfil

$$
\sum_{j=\alpha(p)}^{r} b_{i, j}^{p} q_{j, m}^{0}=\text { known term }
$$

for $m=\alpha(p), \ldots, r$. This system has a solution $\left(b_{i, \alpha(p)}^{p}, \ldots, b_{i, r}^{p}\right)$ since the matrix $\left(\left(q_{j, m}^{0}\right)\right)_{\alpha(p) \leq j, m \leq r}$ is a right-bottom minor of $Q_{k}^{\prime}(0) P^{-1}$.

Corollary 4.1.3 (Plemelj) If there exists $k \in\{1, \ldots, n\}$ such that $\chi\left(\gamma_{k}\right)$ is semi-simple (i.e. diagonalizable) then $\chi$ is the monodromy of a Fuchsian system.

Proof: If $\chi\left(\gamma_{k}\right)$ is semi-simple we can split

$$
\left.H\right|_{U_{k}^{*}}=H_{k, 1} \oplus \ldots \oplus H_{k, r}
$$


into flat line bundles. Let $(E, \nabla)$ be the canonical extension of $H$ (i.e. $\Phi_{j}=0$ for all $j$ ) and choose $Z_{k}$ (see subsection 3.1) such that the i-th section in $Y_{k}\left(\tilde{z}_{k}\right)=Y\left(\tilde{z}_{k}\right) Z_{k}$ spans $H_{k, i}$. Choose $W$ as in Proposition 4.1.2. So $Q_{k}=\hat{Q}_{k} z_{k}^{-C} P$ for invertible $\hat{Q}_{k}$ and permutation $P$, where $C$ is the splitting type of $E$.

Let $P^{\prime}$ be the permutation with $P_{i, j}^{\prime}=0$ if $i+j \neq n$ and $P_{i, n-i}^{\prime}=1$ for $i=1, \ldots, n$. Consider the filtration of $\left.H\right|_{U_{k}^{*}}$, induced by the sections in $Y_{k}\left(\tilde{z}_{k}\right) P^{-1}\left(P^{\prime}\right)^{-1}$. As $\chi\left(\gamma_{k}\right)$ is semi-simple it will respect this filtration. Let $\Phi_{k}^{\prime}:=-\left(P^{\prime}\right)^{-1} C P^{\prime}$, which is diagonal with non-increasing entries, and $\Phi_{j}^{\prime}=0$ for $j \neq k$. Put $\left(E^{\prime}, \nabla^{\prime}\right):=\mathcal{F}\left(H, \Phi_{j}^{\prime}\right)$. Then $W$ trivializes $\left.E^{\prime}\right|_{\mathbb{P}^{1}-x_{k}}$. Furthermore, $Q_{k}^{\prime}$ is invertible and hence, $W$ spans $E^{\prime}$ globally, since

$$
\begin{aligned}
W Q_{k}^{\prime} & =Y_{k}\left(\tilde{z}_{k}\right) P^{-1}\left(P^{\prime}\right)^{-1} \tilde{z}_{k}^{-K_{k}^{\prime}} z_{k}^{-\Phi_{k}^{\prime}}=Y_{k}\left(\tilde{z}_{k}\right) \tilde{z}_{k}^{-K_{k}} P^{-1}\left(P^{\prime}\right)^{-1} z_{k}^{-\Phi_{k}^{\prime}} \\
& =W Q_{k} P^{-1} z_{k}^{C}\left(P^{\prime}\right)^{-1}=W \hat{Q}_{k}\left(P^{\prime}\right)^{-1} .
\end{aligned}
$$

\subsection{The rank two case}

For a representation $\chi: \pi_{1}(S, s) \rightarrow \mathrm{Gl}(2, \mathscr{C})$ with canonical extension $\left(E^{0}, \nabla^{0}\right)=\mathcal{F}(H, 0)$ of splitting type $C^{0}=\operatorname{diag}\left(c_{1}^{0}, c_{2}^{0}\right)$, Bolibruch calls $c_{1}^{0}-c_{2}^{0}$ the weight of the canonical extension, [1, p 102].

Theorem 4.2.1 (i) (Dekkers) Any rank two representation $\chi$ is the holonomy of a Fuchsian system.

(ii) (Bolibruch, [1, p 137]) $c_{1}^{0}-c_{2}^{0}=\min _{\phi} \sum_{1}^{n}\left(\phi_{j}^{1}-\phi_{j}^{2}\right)$ where $\phi$ runs over all integer weight functions on $H$ such that $\mathcal{F}(H, \phi)$ is Fuchsian.

Proof: Assume $(E, \nabla)=\mathcal{F}(H, \phi)$ is Fuchsian. The identity of $H$ extends to a meromorphic map $\mathcal{O}\left(c_{1}^{0}\right) \oplus \mathcal{O}\left(c_{2}^{0}\right)=E^{0} \rightarrow E$. By definition of $\mathcal{F}$ (or the proof of Lemma 2.3.6), the non-zero map $\mathcal{O}\left(c_{1}^{0}\right) \rightarrow E$ is of order greater or equal $\phi_{j}^{2}$ at $x_{j}$. Hence, $c_{1}^{0} \leq-\sum_{1}^{n} \phi_{j}^{2}$. By Proposition 3.1.3, $c_{1}^{0}+c_{2}^{0}+\sum\left(\phi_{j}^{1}+\phi_{j}^{2}\right)=0$ and hence $c_{1}^{0}-c_{2}^{0} \leq \sum_{1}^{n}\left(\phi_{j}^{1}-\phi_{j}^{2}\right)$. This proves (ii) in one direction.

If there exists $k \in\{1, \ldots, n\}$ such that $\chi\left(\gamma_{k}\right)$ has two eigenvalues then we are done by the proof of Corollary 4.1.3. Otherwise, $\left(E^{0}, \nabla^{0}\right)=\mathcal{F}(H, 0)$ is semi-stable and we can argue much as in Theorem 2.3.6. If $v_{i}: \mathbb{P}^{1} \rightarrow \mathcal{O}\left(c_{i}^{0}\right)$ vanishes to order $c_{i}^{0}$ at $s$ and $\pi_{2}: E^{0} \rightarrow \mathcal{O}\left(c_{2}^{0}\right)$, then

$$
\pi_{2} \circ \nabla^{0}\left(v_{1}\right): \mathbb{P}^{1} \rightarrow \mathcal{O}\left(c_{2}^{0}\right) \otimes \mathcal{K}_{\mathbb{P}^{1}} \otimes[X]
$$


either vanishes identically, in which case $c_{1}^{0}=c_{2}^{0}$ and we are done, or it is of order at least $c_{1}^{0}$ at $s$. If it also vanishes at each $x_{j}$ then $n+c_{1}^{0} \leq c_{2}^{0}+n-2$. So, assume $\left(\pi_{2} \circ \nabla^{0}\left(v_{1}\right)\left(x_{k}\right) \neq 0\right.$. If $W=\left(v_{1}, v_{2}\right)$, the formula for the connectionmatrix $\left.\omega\right|_{U_{k}^{*}}$ in the proof of Proposition 3.1.3 implies that $\left(Q_{k}\right)_{1,2}\left(x_{k}\right) \neq 0$ $\left(\operatorname{det} Q_{k}\left(x_{k}\right) \neq 0\right.$ by choice of $W$ ).

Now apply the Claim at the beginning of the proof of Proposition 4.1.2 with $W^{\prime}=\left(v_{1}, v_{2}\right)$ and $P$ the non-trivial rank two permutation. Then $\mathcal{F}\left(H, 0, \ldots, 0, \Phi_{k}, 0, \ldots, 0\right)$ will be Fuchsian for $\Phi_{k}=-P^{-1} C^{0} P$. We have completed the proof of (ii) and also proved (i).

\subsection{The semi-stable case and implications}

Theorem 4.3.1 Let $H \rightarrow S$ be a flat bundle. If we can find filtrations $H_{j}^{m}$ and integer weights $\Phi_{j}$ such that

(a) $\left(H, H_{j}^{m}, \Phi_{j}\right)$ is semi-stable and

(b) there exists $k \in\{1, \ldots, n\}$ with $\operatorname{rank}\left(H_{k}^{i+1} / H_{k}^{i}\right)=1$ and

$$
\phi_{k}^{i}-\phi_{k}^{i+1} \geq(r-1)(n-2) \forall i=1, \ldots, r-1
$$

then we can find $\Phi_{k}^{\prime}$ such that $\left(E^{\prime}, \nabla^{\prime}\right):=\mathcal{F}\left(H, H_{j}^{m}, \Phi_{1}, \ldots, \Phi_{k}^{\prime}, \ldots, \Phi_{n}\right)$ is Fuchsian.

Proof: Let $C$ be the splitting type of $(E, \nabla)=\mathcal{F}\left(H, H_{j}^{m}, \Phi_{j}\right)$. Theorem 3.2.1 implies

$$
c_{1}-c_{r} \leq(n-2)(r-1) \leq \phi_{k}^{i}-\phi_{k}^{i+1}
$$

for all $i=1, \ldots, r-1$. Fix $W$ as in Proposition 4.1.2 and let, in that notation, $\Phi_{k}^{\prime}:=\Phi_{k}-P^{-1} C P$, which will have non-increasing entries. $W$ spans $E^{\prime}$ off $x_{k}$ and

$$
W Q_{k} z_{k}^{\Phi_{k}} \tilde{z}_{k}^{K_{k}}=Y_{k}\left(\tilde{z}_{k}\right)=W Q_{k}^{\prime} z_{k}^{\Phi_{k}^{\prime}} \tilde{z}_{k}^{K_{k}}
$$

implies

$$
Q_{k}^{\prime}=Q_{k} z_{k}^{P^{-1} C P}=\hat{Q}_{k} z_{k}^{-C} P z_{k}^{P^{-1} C P}=\hat{Q}_{k} P .
$$

So, $Q_{k}^{\prime}$ is invertible and $W$ a global trivialisation of $E^{\prime}$. 
Proposition 4.3.2 Suppose there exists $k \in\{1, \ldots, n\}$ and $h \in H_{s}$ such that $h$ is an eigenvector of $\chi\left(\gamma_{k}\right)$ but a cyclic vector of the $\pi_{1}(S, s)$-module $H_{s}$ (i.e. $\left.\langle(\operatorname{Im} \chi)(h)\rangle=H_{s}\right)$. Let $N_{1}, \ldots, N_{n}$ be any integers.

Then we can find filtrations $H_{j}^{m}$ and weights $\Phi_{j}$ such that $\mathcal{F}\left(H, H_{j}^{m}, \Phi_{j}\right)$ is Fuchsian. Moreover, we can arrange that

(1) $\phi_{j}^{i} \geq N_{j}$ for all $j \neq k, i=1, \ldots, r$ and

(2) $\phi_{k}^{1}=\phi(h) \geq N_{k}$.

Hence, there are infinitely many Fuchsian systems with monodromy $\chi$.

Proof: For each $j \neq k$ choose filtrations $H_{j}^{m}$ and weights $\Phi_{j}$ such that (1) is satisfied. Also choose a filtration $H_{k}^{m}$ and weights $\Phi_{k}$ such that $H_{k}^{1}=\langle h\rangle$, hypothesis (b) of Theorem 4.3 .1 is satisfied and $\phi_{k}^{1} \geq N_{k}+(r-1)(n-2)$. These conditions remain satisfied if we increase $\phi_{k}^{1}$ or decrease $\phi_{k}^{r}$. Doing so we can assume that $\operatorname{deg}\left(H, H_{j}^{m}, \Phi_{j}\right)=0$ and since no proper flat subbundle of $H$ contains $h$ we can also assume that $\left(H, H_{j}^{m}, \Phi_{j}\right)$ is semi-stable.

Apply Theorem 4.3.1 to find $\sum_{1}^{r} c_{i}=0$ and $c_{i}-c_{i+1} \leq(n-2)$, implying $\left|c_{i}\right| \leq(n-2)(r-1)$ for $i=1, \ldots, r$. Hence, the first entry of $\Phi_{k}^{\prime}=\Phi_{k}-P^{-1} C P$ is greater or equal to $N_{k}+(r-1)(n-2)-(r-1)(n-2)=N_{k}$ and we are done.

Corollary 4.3.3 (Bolibruch, [⿴囗十, p 84], [1, p 83]; Kostov, [14]) Any irreducible flat $H \rightarrow S$ is the restriction of a Fuchsian system.

Corollary 4.3.4 ([1, p 114]) Any $\chi: \pi_{1}(S, s) \rightarrow G l(r, \mathscr{C})$ is the subrepresentation of the monodromy of some Fuchsian system of double the rank.

Proof: By Lemma 4.1.1 we may assume that $n \geq 3$ and $r \geq 2$. Let $G_{j}:=\chi\left(\gamma_{j}\right)$ for all $j=1, \ldots, n$. By Corollary 4.1 .3 we can assume that in canonical basis $e_{i}=(0, \ldots, 0,1,0, \ldots, 0)^{t}$ we have the equality of vector spaces $\left\langle e_{r}, G_{1} e_{r}\right\rangle=\left\langle e_{r-1}, e_{r}\right\rangle$. We define $G_{j}^{\prime}$ as follows

$$
G_{1}^{\prime}:=\left(\begin{array}{cc}
G_{1} & M_{1} \\
0 & I
\end{array}\right) \quad M_{1}:=\left(\begin{array}{cccccc}
1 & & & & & \\
& \cdot & 0 & & & \\
& 0 & \cdot & & & \\
& & & 1 & & \\
& & & & 0 & 0 \\
& & & & 1 & 0
\end{array}\right)
$$




$$
\begin{array}{r}
G_{2}^{\prime}:=\left(\begin{array}{cc}
G_{2} & 0 \\
0 & M_{2}
\end{array}\right) \quad M_{2}:=\left(\begin{array}{ccccc}
1 & 1 & & & \\
& \cdot & \cdot & 0 & \\
& & \cdot & \cdot & \\
& 0 & & \cdot & 1 \\
& & & 1
\end{array}\right) \\
G_{3}^{\prime}:=\left(\begin{array}{cc}
G_{3} & -G_{2}^{-1} G_{1}^{-1} M_{1} \\
0 & M_{2}^{-1}
\end{array}\right) \text { and } G_{j}^{\prime}:=\left(\begin{array}{ccc}
G_{j} & 0 \\
0 & I
\end{array}\right)
\end{array}
$$

for all $j \geq 4$. One checks that $G_{1}^{\prime} \cdot \ldots \cdot G_{n}^{\prime}=I_{2 r}$ and so defines a representation $\chi^{\prime}: \pi_{1}(S, s) \rightarrow \operatorname{Gl}(2 r, \mathscr{C})$. Furthermore, $e_{2 r}$ is an eigenvector of $G_{1}^{\prime}=\chi^{\prime}\left(\gamma_{1}\right)$ and

$$
\begin{gathered}
\left\langle e_{2 r}, G_{2}^{\prime} e_{2 r}, \ldots,\left(G_{2}^{\prime}\right)^{r-1} e_{2 r}\right\rangle=\left\langle e_{r+1}, \ldots, e_{2 r}\right\rangle, \\
G_{1}^{\prime}\left\langle e_{r+1}, \ldots, e_{2 r}\right\rangle \oplus\left\langle e_{r+1}, \ldots, e_{2 r}\right\rangle \supset\left\langle e_{1}, \ldots, e_{r-2}, e_{r}, \ldots, e_{2 r}\right\rangle
\end{gathered}
$$

and $\left\langle e_{r}, G_{1}^{\prime} e_{r}\right\rangle=\left\langle e_{r-1}, e_{r}\right\rangle$. Apply Proposition 4.3.2 with $h:=e_{2 r}$.

\subsection{Reducible representations}

Let $H_{j}:=\left.H\right|_{U_{j}^{*}}$. Part (i) of the following is due to Bolibruch, [1, Cor. 5.4.1], [4, Thm. 3.8], while (ii) is new and will be used to give an alternative proof of Bolibruch's answer to the RHP in rank three, Theorem 4.5.3 here.

Theorem 4.4.1 Let $H^{\prime} \rightarrow H \rightarrow H^{\prime \prime}$ be a short exact sequence of flat bundles (without weights) and assume there exist filtrations and weights such that $\left(E^{\prime \prime}, \nabla^{\prime \prime}\right)=\mathcal{F}\left(H^{\prime \prime},\left(H^{\prime \prime}\right)_{j}^{m}, \Phi_{j}^{\prime \prime}\right)$ is Fuchsian. Suppose at least one of the following conditions holds for some $k \in\{1, \ldots, n\}$.

(i) $H_{k}^{\prime} \rightarrow H_{k} \rightarrow H_{k}^{\prime \prime}$ splits and there exist filtrations and weights such that $\left(E^{\prime}, \nabla^{\prime}\right)=\mathcal{F}\left(H^{\prime},\left(H^{\prime}\right)_{j}^{m}, \Phi_{j}^{\prime}\right)$ is Fuchsian.

(ii) There exist splittings $H_{k}^{\prime}=H^{(3)} \oplus H^{(0)}$ and $H_{k}=H^{(3)} \oplus H^{(4)}$ where $\langle h\rangle=H^{(0)}=H_{k}^{\prime} \cap H^{(4)}$ and $h$ is a cyclic vector of the $\pi_{1}(S, s)$ module $H_{s}^{\prime}$.

Then $H$ is the restriction of a Fuchsian system.

Proof: (i): By Lemma 3.2.3 we can assume that for all $j \neq k$ the smallest diagonal entry in $\Phi_{j}^{\prime}$ is greater then the largest one in $\Phi_{j}^{\prime \prime}$. Using

$$
H_{j}^{\prime} \rightarrow H_{j} \rightarrow H_{j}^{\prime \prime}
$$


for such $j$ we can therefore induce filtrations and weights on $H_{j}$ to make this local sequence a short exact one of weighted flat bundle.

Let $\alpha: H_{k}^{\prime \prime} \rightarrow H_{k}$ be a splitting right inverse of $H_{k} \rightarrow H_{k}^{\prime \prime}$ and put the obvious weighted filtration on $\operatorname{Im} \alpha$. Then use $H_{k}=H_{k}^{\prime} \oplus \operatorname{Im} \alpha$ to give $H_{k}$ the direct sum weighted filtration. $\left(H_{k}^{\prime}, \phi_{k}^{\prime}\right) \rightarrow\left(H_{k}, \phi_{k}\right)$ becomes an injection. Since $\left(H_{k}, \phi_{k}\right) \rightarrow\left(H_{k}^{\prime \prime}, \phi_{k}^{\prime \prime}\right)$ is the composition $H_{k} \rightarrow \operatorname{Im} \alpha \stackrel{\alpha^{-1}}{\rightarrow} H_{k}^{\prime \prime}$, it is a surjection. Apply Lemma 3.2.4 to finish this case.

(ii): For $j=1, \ldots, n$ let $N_{j}$ be the greatest diagonal entry in $\Phi_{j}^{\prime \prime}$. Then construct $\left(H^{\prime},\left(H^{\prime}\right)_{j}^{m}, \Phi_{j}^{\prime}\right)$ as in Proposition 4.3 .2 so that $\mathcal{F}\left(H^{\prime},\left(H^{\prime}\right)_{j}^{m}, \Phi_{j}^{\prime}\right)$ is Fuchsian. If $j \neq k$ we induce weights on $H_{j}$ as in (i).

Use the exact sequence of flat bundles $H^{(0)} \rightarrow H^{(4)} \rightarrow H_{k}^{\prime \prime}$ to induce weights $\phi^{(4)}$ on $H^{(4)}$. Induce weights on $H_{k}$ using $H_{k}=H^{(3)} \oplus H^{(4)}$. Then, $\left(H_{k}, \phi_{k}\right) \rightarrow\left(H_{k}^{\prime \prime}, \phi_{k}^{\prime \prime}\right)$ is given by the composition

$$
\left(H_{k}, \phi_{k}\right) \rightarrow\left(H^{(4)}, \phi^{(4)}\right) \rightarrow\left(H_{k}^{\prime \prime}, \phi_{k}^{\prime \prime}\right)
$$

of two surjections and hence is one itself. For $h^{\prime}=h^{(3)}+h^{(0)} \in H_{k}^{\prime}$ we have

$$
\phi_{k}^{\prime}\left(h^{\prime}\right)=\min \left(\phi_{k}^{\prime}\left(h^{(3)}\right), \phi_{k}^{\prime}\left(h^{(0)}\right)\right)=\phi\left(h^{\prime}\right)
$$

since $H^{(0)}$ is the highest weight subspace in the filtration of $H_{k}^{\prime}$. Hence $\left(H_{k}^{\prime}, \phi_{k}^{\prime}\right) \rightarrow\left(H_{k}, \phi_{k}\right)$ is an injection and we finish with Lemma 3.2.4.

Proposition 4.4.2 ([4, p 83], [1, p 100]) If $H$ is reducible, the holonomy $\chi\left(\gamma_{j}\right)$ has only one Jordan-block, for each $j=1, \ldots, n$, and $\mathcal{F}(H, \phi)=$ $(E, \nabla)$ has constant splitting type, then $\Phi_{j}=\phi_{j}^{1} \cdot I_{r}$ for all $j$.

We give a more conceptual proof.

Proof: If $G_{j}$ has only one Jordan block, there exists a canonical full flag of subsystems of $H_{j}$. If $H^{\prime}$ is a proper subsystem of $H$ then it must contain the subbundles in the local filtrations of rank equal to rank $H^{\prime}$. If some $\Phi_{k}$ has non equal diagonal entries then

$$
\text { slope }\left(H^{\prime}, \phi^{\prime}\right)>\text { slope }(H, \phi) \text {. }
$$

But $(E, \nabla)$, and hence $(H, \phi)$, is semi-stable - a contradiction.

Let $H$ be as in the previous proposition, $\rho_{j}$ the only eigenvalue of $\chi\left(\gamma_{j}\right)$ and $\mu_{j}=\operatorname{norm} \log \rho_{j}$. If $(E, \nabla)=\mathcal{F}(H, \phi)$ is Fuchsian then $\operatorname{deg}(H, \phi)=0$. 
Since $r \mid \sum \operatorname{Tr} \Phi_{j}$ we find $r \mid \sum \operatorname{Tr}\left(\right.$ norm $\left.\log \chi\left(\gamma_{j}\right)\right)$, i.e. $r \mid r \sum \mu_{j}$. So, $\sum \mu_{j}$ must be an integer. Bolibruch uses this to give an example of a representation with $r=4$ and $n=3$ which can not be the monodromy of any Fuchsian system with three singularities, [4, p 91], [1, p 105].

\subsection{Parabolic representations and the rank three case}

Let B $(r, \mathscr{C})$ be the group of invertible upper-triangular $r \times r$-matrices.

Theorem 4.5.1 Let $\chi: \pi_{1}(S, s) \rightarrow B(r, \mathbb{C})$ be a representation with

$$
G_{j}=\chi\left(\gamma_{j}\right)=\left(\begin{array}{ccccc}
\rho_{j}^{1} & & & \\
& \cdot & \star & \\
& 0 & \cdot & \\
& & & \rho_{j}^{r}
\end{array}\right)
$$

for $j=1, \ldots, n$. Let $\mu_{j}^{i}:=\operatorname{norm} \log \left(\rho_{j}^{i}\right)$ and $\Lambda^{i}:=-\sum_{j=1}^{n} \operatorname{Re} \mu_{j}^{i} \in \mathbb{Z}_{\leq 0}$. Assume we can find $\left(\left(\phi_{j}^{i}\right)\right)$ such that

(a) $\phi_{j}^{i} \geq \phi_{j}^{k}$ if $\left(i \leq k\right.$ and $\left.\rho_{j}^{i}=\rho_{j}^{k}\right)$ and

(b) $\Lambda^{i}=\sum_{j=1}^{n} \phi_{j}^{i}$ for all $i=1, \ldots, r$.

Then $\chi$ is the monodromy of a Fuchsian system $(E, \nabla)$ and the integer weights of $(E, \nabla)$ equal $\left(\left(\phi_{j}^{i}\right)\right)$ as sets.

Proof: The flat bundle $H$, associated to $\chi$, has a global natural filtration. We work by induction on the rank $r$ and extend the claim of the theorem by the fact that the integer weights function $\phi_{j}$ on $H_{j}$, which we construct, is the direct sum of its restrictions to the generalised eigenspaces of $G_{j}$, acting on $H_{j}$.

For $r=1$ we let $\phi_{j}: H_{j}-\{0\} \rightarrow \mathbb{Z}$ have single value $\phi_{j}^{1}$. This implies $\operatorname{deg}\left(H, H_{j}^{m}, \Phi_{j}\right)=\Lambda^{1}-\Lambda^{1}=0$ and hence $\mathcal{F}\left(H, H_{j}^{m}, \Phi_{j}\right)$ is Fuchsian.

For $r \geq 2$ write $H^{\prime} \subseteq H, H_{j}^{\prime} \subseteq H_{j}$ for the rank $(r-1)$ subbundles. We are given, by induction,

$$
\phi_{j}^{\prime}: H_{j}^{\prime} \rightarrow\left\{\phi_{j}^{1}, \ldots, \phi_{j}^{r-1}\right\} \cup\{+\infty\}
$$

with the above described property. Consider one $j \in\{1, \ldots, n\}$ at a time. Let $A \subseteq H_{j}\left(A^{\prime} \subseteq H_{j}^{\prime}\right)$ be the generalised eigenspace of $G_{j}\left(G_{j}^{\prime}\right)$ of the eigenvalue $\rho_{j}^{r}$. Also let $B^{\prime} \subseteq H_{j}^{\prime}$ be the direct sum of the other generalised eigenspaces 
of $G_{j}^{\prime}$. Then, the extended induction hypothesis implies $\phi_{j}^{\prime}\left(h_{A^{\prime}}+h_{B^{\prime}}\right)=$ $\left.\min \left(\phi_{j}^{\prime}\left(h_{A^{\prime}}\right), \phi_{j}^{\prime}\right)\left(h_{B^{\prime}}\right)\right)$. Put

$$
\begin{aligned}
\phi_{A}: A & \rightarrow \mathbb{Z} \cup\{+\infty\} \\
h & \mapsto\left\{\begin{array}{ccc}
\phi_{j}^{\prime}(h) & \text { if } & h \in A^{\prime} \\
\phi_{j}^{r} & \text { if } & h \in A-A^{\prime} .
\end{array}\right.
\end{aligned}
$$

We give $H_{j}=A \oplus B^{\prime}$ the direct sum of the weighted filtrations. By construction, $\left(H_{j}^{\prime}, \phi_{j}^{\prime}\right) \rightarrow\left(H_{j}, \phi_{j}\right)$ is an injection. We give $H_{j} / H_{j}^{\prime}$ the integer weight $\phi_{j}^{r}$. Then $h=h_{A}+h_{B^{\prime}} \in H_{j}$ maps to zero under $\alpha: H_{j} \rightarrow H_{j} / H_{j}^{\prime}$ unless $h_{A} \in A-A^{\prime}$ in which case

$$
\phi(h)=\min \left(\phi_{A}\left(h_{A}\right), \phi_{j}^{\prime}\left(h_{B^{\prime}}\right)\right) \leq \phi_{A}\left(h_{A}\right)=\phi_{j}^{r} .
$$

$\alpha$ is surjective since $\phi\left(h_{A}\right)=\phi_{j}^{r}$ for any $h_{A} \in A-A^{\prime}$. We have constructed a short exact sequence

$$
\left(H^{\prime}, \phi^{\prime}\right) \rightarrow(H, \phi) \rightarrow\left(H / H^{\prime}, \phi^{H^{\prime} / H}\right)
$$

where $H^{\prime}$ is the restriction of a Fuchsian system by induction and $H / H^{\prime}$ is so similar to the rank one case. Apply Lemma 3.2.4 to finish.

The following result is due to Bolibruch when $r=3$, [1, p 133]. He has a counter example for $r=7, n=4$, [1, p 106].

Corollary 4.5.2 For $\chi: \pi_{1}(S, s) \rightarrow B(r, \mathbb{C})$ and $r \in\{1,2,3,4\}$ there exists a Fuchsian system with monodromy $\chi$.

Proof: We want to find $\left(\left(\phi_{j}^{i}\right)\right)$ satisfying (b) of Theorem 1.5.1 and (a)': $\phi_{j}^{i}=\phi_{j}^{k}$ if $\rho_{j}^{i}=\rho_{j}^{k}$.

Claim : If there is $m \in\{1, \ldots, n\}$ and $k \in\{1, \ldots, r\}$ such that $\rho_{m}^{k} \neq \rho_{m}^{t}$ for all $t \neq k$ then the problem to find $\left(\left(\phi_{j}^{i}\right)\right)$, satisfying (b) and (a)', reduces to rank $(r-1)$.

To see this just find $\left(\left(\phi_{j}^{i}\right)\right)_{i \neq k}$, choose $\left(\phi_{j}^{k}\right)_{j \neq m}$ if they are not fixed by (a)' already and calculate $\phi_{m}^{k}$ using (b).

The corollary is trivial for $r=1$. For $r=2,3$, we can either use the claim or have $\Lambda^{1}=\ldots=\Lambda^{r}$ and finish easily. For $r=4$, if we can not use the claim there are two cases. Either, for each $j=1, \ldots, n,\left(\rho_{j}^{1}=\rho_{j}^{3}\right.$ and $\left.\rho_{j}^{2}=\rho_{j}^{4}\right)$ or $\left(\rho_{j}^{1}=\rho_{j}^{4}\right.$ and $\left.\rho_{j}^{2}=\rho_{j}^{3}\right)$. Hence, $\Lambda^{1}+\Lambda^{2}-\Lambda^{3}=\Lambda^{4}$. Solve the 
system consisting of (a)' and (b) for $\left(\left(\phi_{j}^{i}\right)\right)_{j=1, . ., n ; i=1,2,3}$ and get a solution for the rank four problem by setting $\phi_{j}^{4}:=\phi_{j}^{1}+\phi_{j}^{2}-\phi_{j}^{3}$ for $j=1, \ldots, n$.

Or, $\rho_{m}^{1}=\rho_{m}^{2} \neq \rho_{m}^{3}=\rho_{m}^{4}$ for some $m \in\{1, \ldots, n\}$. Solve two rank two problems, i.e. find $\left(\left(\phi_{j}^{i}\right)\right)_{i=1,2}$ satisfying (a)' and (b) and find $\left(\left(\phi_{j}^{i}\right)\right)_{i=3,4}$ satisfying (a)' and (b). Increasing all entries of $\left(\left(\phi_{j}^{i}\right)\right)_{i=1,2 ; j \neq m}$ by a sufficiently large integer $N$ and decreasing $\phi_{m}^{1}$ and $\phi_{m}^{2}$ by $(n-1) N$, we can satisfy (a) and (b) for the rank four problem.

Theorem 4.5.3 ([4, p 90], [1, p 133]) A rank three representation $\chi$ : $\pi_{1}(S, s) \rightarrow G l(3, \mathscr{C})$ is the monodromy of a Fuchsian system if and only if one or more of the following holds.

(a) $\chi$ is irreducible.

(b) Some $\chi\left(\gamma_{k}\right)=G_{k}$ has more than one Jordan block.

(c) The canonical extension of $H=H(\chi)$ has constant splitting type.

The part of the proof which is left we do differently from Bolibruch.

Proof: By Corollary 4.3.3, Corollary 4.1.3, Lemma 3.2.3 and Proposition 4.4 .2 we are left to show that if $\chi\left(\gamma_{k}\right)$ has two Jordan blocks for some $k$ then $\chi$ is the monodromy of a logarithmic connection on $\mathbb{P}^{1} \times \mathbb{C}^{3}$.

Let $h_{1} \in \mathbb{C}^{3}\left(h_{2} \in \mathbb{C}^{3}\right)$ be the eigenvector corresponding to the rank one (rank two) Jordan block of $\chi\left(\gamma_{k}\right)$. Consider the $\pi_{1}(S, s)$-submodules

$$
F_{1}:=\left\langle(\operatorname{Im} \chi)\left(h_{1}\right)\right\rangle \text { and } F_{2}:=\left\langle(\operatorname{Im} \chi)\left(h_{2}\right)\right\rangle
$$

of $\mathbb{C}^{3}$. If rank $F_{1}=1$ we are in case (i) of Theorem 4.4.1 because of the positive solvability of the RHP in rank two. If $\operatorname{rank} F_{1}=3$ then we are in the case of Proposition 4.3.2. So assume rank $F_{1}=2$ and hence $h_{2} \in F_{2} \subseteq F_{1}$ and $\operatorname{rank} F_{2}<3$. If rank $F_{2}=1$ we are in the case of Corollay 4.5 .2 and if $\operatorname{rank} F_{2}=2$ we are in case (ii) of Theorem 4.4.1 with $H^{\prime}=F_{1}, H^{(0)}=\left\langle h_{2}\right\rangle$ and $H^{(3)}=\left\langle h_{1}\right\rangle$.

\section{References}

[1] D. V. Anosov, A. A. Bolibruch, "The Riemann-Hilbert Problem," Aspects of Mathematics E22, Vieweg \& Sohn Verlagsgesellschaft mbH, Braunschweig/Wiesbaden, 1994. 
[2] M. F. Atiyah, Complex analytic connections in fibre bundles, Trans. Amer. Math. Soc. 85 (1957), 181-207.

[3] A. Beauville, Monodromie des systèmes différentiels linéaires à pôles simples sur la sphère de Riemann, Séminaire Bourbaki 45 (1992-93), 765-01 - 765-15.

[4] A. A. Bolibruch, Hilbert's twenty-first problem for Fuchsian linear systems, in "Developments in Mathematics: The Moscow School," ed V. Arnold, M. Monastyrsky, Chapman \& Hall, London , 1993.

[5] W. DekKers, The matrix of a connection having regular singularities on a vector bundle of rank 2 on $\mathbb{P}^{1}(\mathscr{C})$, in "Lecture Notes in Mathematics 712," Springer, Berlin, 1979, 33-43.

[6] P. Deligne, Equations Différentielles à Points Singuliers Réguliers, in "Lecture Notes in Mathematics 163," Springer, Berlin, 1970.

[7] H. Esnault, E. Viehweg, Logarithmic de Rham complexes and vanishing theorems, Inv. Math. 86 (1986), 161-194.

[8] O. Forster, "Lectures on Riemann Surfaces," Springer, New York, 1981.

[9] F. R. Gantmacher, "Applications of the Theory of Matrices," Interscience Publishers, New York, 1959.

[10] P. Griffiths, J. Harris, "Principles of Algebraic Geometry," John Wiley \& Son's, New York, 1978.

[11] R. M. Hain, On a generalisation of Hilbert's 21st problem, Ann. scient. Ec. Norm. Sup. 419 (1986), 609-627.

[12] D. Hilbert, Mathematische Probleme, Nachr. Ges. Wiss. Göttingen (1900), 253-297.

[13] S. Kobayashi, "Differential geometry of complex vector bundles," Iwanami Shoten and Princeton University Press, Princeton, 1987.

[14] V. P. Kostov, Fuchsian linear systems on $\mathbb{C} \mathbb{P}^{1}$ and RiemannHilbert's Problem, preprint 674, University of Utrecht, 1991.

[15] A. H. M. Levelt, Hypergeometric functions, Nederl. Akad. Wetensch. Proc. A 64 (1961), 361-403. 
[16] Y. Manin, Moduli Fuchsiani, Ann. Scu. Norm. Sup. Pisa (III) 19 (1965), 113-126.

[17] H. J. NAstold, Über meromorphe Schnitte komplex analytischer Vektorraumbündel and Anwendungen auf Riemannsche Klassen, Math. Z. 70 (1958), 55-92.

[18] C. Okonek, M. Schneider, H. Spindler, "Vector Bundles on Complex Projective Spaces," Birkhäuser, Boston, 1980.

[19] J. PlemelJ, "Problems in the sense of Riemann and Klein," Interscience, New York, 1964.

[20] H. RöHRL, Das Riemann-Hilbertsche Problem der Theorie der linearen Differentialgleichungen, Math. Ann. 133 (1957), 1-25.

[21] C. T. Simpson, Harmonic bundles on noncompact curves, J. Amer. Math. Soc. (3) 3 (1990), 713-770. 